\title{
Increase of programmed death-1-expressing intratumoral CD8 T cells predicts a poor prognosis for nasopharyngeal carcinoma
}

\author{
Mei-Chi Hsu ${ }^{1,7}$, Jenn-Ren $\mathrm{Hsiao}^{2,3,7}$, Kung-Chao Chang ${ }^{4,7}$, Yuan-Hua Wu ${ }^{3,5}$, Ih-Jen Su ${ }^{1,4}$, \\ Ying-Tai Jin ${ }^{4}$ and Yao Chang ${ }^{1,6}$
}

${ }^{1}$ Division of Infectious Diseases, National Health Research Institutes, Tainan, Taiwan, ROC; ${ }^{2}$ Department of Otolaryngology, Medical College and Hospital, National Cheng Kung University, Tainan, Taiwan, ROC; ${ }^{3} H e a d$ and Neck Collaborative Oncology Group of Cancer Center, Medical College and Hospital, National Cheng Kung University, Tainan, Taiwan, ROC; ${ }^{4}$ Department of Pathology, Medical College and Hospital, National Cheng Kung University, Tainan, Taiwan, ROC; ${ }^{5}$ Department of Radiation Oncology, Medical College and Hospital, National Cheng Kung University, Tainan, Taiwan, ROC and ${ }^{6}$ Department of Microbiology and Immunology, Medical College and Hospital, National Cheng Kung University, Tainan, Taiwan, ROC

\begin{abstract}
Intratumoral cytotoxic T lymphocytes are critical for controlling tumor recurrence, and programmed death-1 (PD-1) is a recognized marker of T-cell dysfunction. We analyzed this marker and its binding ligands in nasopharyngeal tumor tissue and non-cancerous nasopharyngeal control tissue to retrospectively evaluate the correlation between its expression and the post-treatment outcome of nasopharyngeal carcinoma patients. Using double immunofluorescence staining, we found that the expression of PD-1 in CD8 T cells in tumor tissue was significantly higher than in control tissue (mean: 28.4 vs $3.9 \%, P<0.0001$ ). Although the expression rate of PD-1 in intratumoral CD8 cells was not associated with the other clinicopathological parameters examined, the higher expression rate in this subset of $\mathrm{T}$ cells significantly correlated with a poorer prognosis of overall survival, disease-free survival, and locoregional recurrence-free survival of the cancer patients $(P=0.05,0.007$, and 0.004, respectively). Multivariate analysis confirmed it as an independent risk factor for death, treatment failure, and local recurrence of nasopharyngeal carcinoma. On the other hand, the expression of PD-1 in CD4 T cells and of its ligands in epithelial and stromal cells was not significantly different between tumor and control tissue, and its expression was not associated with clinical outcome of the cancer patients. We propose that PD-1 expression in CD8 cells reflects the selective suppression of cytotoxic lymphocytes in the tumor microenvironment and predicts recurrence of nasopharyngeal carcinoma after conventional therapies. Modern Pathology (2010) 23, 1393-1403; doi:10.1038/modpathol.2010.130; published online 23 July 2010
\end{abstract}

Keywords: immunofluorescence; intratumoral lymphocyte; nasopharyngeal carcinoma; prognosis; programmed death-1

Nasopharyngeal carcinoma is distinguished from other head-and-neck epithelial cancers by its epidemiological and pathological features and its clinical presentation, including the unique distribution of its endemic areas (southern China,

Correspondence: Dr Y Chang, PhD, Division of Infectious Diseases, National Health Research Institutes, No. 367, Sheng-Li Road, Tainan 704, Taiwan, ROC.

E-mail: yaochang@nhri.org.tw

${ }^{7}$ These authors contributed equally to this work.

Received 12 February 2010; revised 22 June 2010; accepted 23 June 2010; published online 23 July 2010 southeast Asia, the Arctic, and North Africa), close association with Epstein-Barr virus infection, poor differentiation of tumor cells, and high susceptibility to radiotherapy and chemotherapy. ${ }^{1}$ Although radiotherapy alone or combined with chemotherapy is relatively successful for curing the cancer, a substantial proportion of patients do not benefit from these conventional treatments, but show locoregional or distant recurrence. ${ }^{1,2}$ To develop more effective therapies for refractory nasopharyngeal carcinoma, we must be able to identify early those patients who urgently require advanced therapies. $^{2,3}$ 
Recent studies have underlined that effectiveness of radiotherapy and chemotherapy can be influenced by anti-tumor immune responses., ${ }^{4,5}$ After conventional cancer treatments that massively destroy tumor cells, activated immune cells such as cytotoxic tumor-infiltrating lymphocytes (TILs) are important for eliminating residual cancer cells and reducing their recurrence. Nevertheless, multiple immunosuppressive mechanisms in tumor microenvironments can inactivate TILs, which may increase the risk of tumor recurrence after radiotherapy and chemotherapy., ${ }^{4,6}$ A marker indicating TIL dysfunction and predicting a poor outcome after conventional treatment would help identify patients refractory nasopharyngeal carcinoma.

Programmed death-1 (PD-1) is a surface receptor that is expressed by lymphocytes and inhibits their proliferation and effector functions after it binds with PD-1 ligands such as B7-H1 (also known as PD-L1) and B7-DC (also known as PD-L2). ${ }^{7,8} \mathrm{PD}-1$ is a significant marker for $\mathrm{T}$ lymphocytes malfunctioning in response to viral infection. ${ }^{9-12}$ Recently, elevated PD-1 expression in intratumoral lymphocytes and its association with the functional impairment of TILs have also been reported in melanoma, hepatocellular carcinoma, and Hodgkin lymphoma. ${ }^{13-15}$ However, reports on the prognostic value of PD-1 expression for cancer therapy, analyzed in only a few studies, are inconsistent. Increased PD-1 expression in intratumoral immune cells correlates with a poor clinical outcome in renal cell carcinoma and classical Hodgkin lymphoma, but is associated with improved overall survival in follicular lymphoma; ${ }^{16-18}$ however, it is not significantly associated with the prognosis of pancreatic cancer. ${ }^{19}$ These inconsistent results may be attributable to two possibilities that have not been clearly addressed. First, PD-1 expression in different subsets of tumorinfiltrating immune cells may have differential effects on clinical outcome. Second, the functional suppression of PD-1-expressing TILs and its prognostic relevance may depend on whether the ligands of PD-1 are present in the tumor tissue. Therefore, we examined the expression of PD-1 and its ligands in biopsy specimens of nasopharyngeal carcinoma and non-cancerous nasopharyngeal control tissue to determine their correlation with clinical outcome after therapies.

\section{Materials and methods}

\section{Patients and Biopsy Specimens}

Biopsy specimens and clinical data were collected from the Surgical Pathology Laboratory of National Cheng Kung University Hospital. The study protocol, specimen usage, and data retrieval were approved by the Institutional Human Experiment and Ethics Committee of National Cheng Kung
University Hospital (approval number ER-95-107). We retrospectively reviewed the charts of nasopharyngeal carcinoma patients whose pre-treatment tumor biopsies, clinicopathological data, and follow-up records were available, who were free of distant metastasis at diagnosis, and who had completed curative radiotherapy with or without chemotherapy at National Cheng Kung University Hospital during 2003 and 2004. We obtained formalin-fixed, paraffin-embedded tissue blocks from 46 primary tumor biopsies, and the control specimens were non-cancerous nasopharyngeal tissue samples from 22 age-matched patients diagnosed with lymphoid hyperplasia. From a second group of patients, we also obtained snap-frozen tissue specimens from 28 primary tumor biopsies and 29 control nasopharyngeal tissue specimens of lymphoid hyperplasia. Histologically, all the tumors were classified as World Health Organization (WHO) type 2 or 3 , showing a poorly differentiated or undifferentiated phenotype and containing a large number of infiltrating lymphocytes. The age of the cancer patients ranged from 28 to 77, with a median of 51. Among the 74 cancer patients studied (46 with paraffined specimens and 28 with frozen specimens), 23 patients had locoregional relapse or distant metastasis and a total of 14 patients died during the follow-up.

\section{Immunofluorescence Staining}

As the antibodies used in this study were suitable for immunostaining on different tissue sections, immunofluorescence co-staining of PD-1 and T-cell markers (CD8 and CD4) was performed using paraffined tissue sections, and co-staining of the PD-1 ligands (B7-H1 and B7-DC) and an epithelial cell adhesion molecule (EpCAM) was performed using frozen tissue sections. Paraffined sections (3- $\mu \mathrm{m}$ thick) of tumor and control tissues were laid on silanized slides (DakoCytomation, Carpinteria, CA, USA), deparaffinized, rehydrated in phosphatebuffered saline (PBS), and then autoclaved in ethylene diamine tetraacetic acid buffer ( $\mathrm{pH}$ 8.0) for $10 \mathrm{~min}$ to retrieve the antigens. The slides were co-incubated with a goat polyclonal antibody that recognizes human PD-1 (diluted 1:15) (R\&D Systems, Minneapolis, MN, USA) and mouse monoclonal antibodies against human CD8 (clone C8/ 144B, ready-to-use; DakoCytomation) or human CD4 (clone 4B12, 1:30 dilution; Novocastra Laboratories, Newcastle upon Tyne, UK) at room temperature for $1 \mathrm{~h}$. After they had been washed three times with PBS, the slides were co-incubated with a fluorescein isothiocyanate (FITC)-conjugated rabbit anti-goat antibody (DakoCytomation) and a tetramethylrhodamine isothiocyanate (TRITC)-conjugated rabbit anti-mouse antibody (DakoCytomation) in the dark at room temperature for $1 \mathrm{~h}$. In the final step, the slides were washed with PBS, mounted with 
a mounting medium (Vector Laboratories, Burlingame, CA, USA), and then examined under a fluorescence microscope (Olympus, Tokyo, Japan) by a pathologist blinded to the clinical information of the patients. Cells that stained positive for PD-1 were green (FITC), and those that stained positive for CD4 and CD8 were red (TRITC). To analyze the expression rates of PD-1 in CD8 and CD4 cells, randomly selected micrographs of the double-staining results were digitally captured and merged, and 800 cells from each sample were manually counted to quantify the percentage of $\mathrm{PD}-1$-positive $\left(\mathrm{PD}-1^{+}\right)$ CD8 and CD4 cells.

To detect PD-1 ligands and EpCAM, cryostat sections (5- $\mu \mathrm{m}$ thick) of tumor and control tissue were fixed in acetone, rehydrated in PBS for $5 \mathrm{~min}$, and then co-incubated in the dark at room temperature for $1 \mathrm{~h}$ with an FITC-conjugated monoclonal antibody that recognizes human EpCAM (1:10 dilution) (clone HEA-125; Miltenyi Biotec, Bergisch Gladbach, Germany) and with phycoerythrinconjugated monoclonal antibodies against human B7-H1 (clone MIH1, ready-to-use; eBioscience, San Diego, CA, USA) or human B7-DC (clone MIH18, ready-to-use; eBioscience). After they had been washed three times with PBS, the sections were then co-incubated in the dark at room temperature for 30 min with a biotinylated rabbit anti-phycoerythrin antibody (1:100 dilution) (eBioscience) and an Alexa488-conjugated rabbit anti-FITC antibody (1:100 dilution) (Invitrogen, Carlsbad, CA, USA). After the specimens had been washed three more times with PBS, the sections were reacted with Alexa594-conjugated streptavidin (1:500 dilution) (Invitrogen) in the dark at room temperature for $30 \mathrm{~min}$. Finally, the sections were washed again, mounted, and examined under a fluorescence microscope. Cells that stained positive for EpCAM were green (FITC plus Alexa488), and those that stained positive for B7-H1 and B7-DC were red (phycoerythrin plus Alexa594). Micrographs of the double-staining results were digitally captured and merged to evaluate whether the ligands of PD-1 were expressed by EpCAM-positive $\left(\mathrm{EpCAM}^{+}\right)$ epithelial cells or EpCAM-negative $\left(\mathrm{EpCAM}^{-}\right)$ stromal cells.

\section{Statistical Analysis}

Student's $t$-test was used to evaluate the differences between $\mathrm{PD}-1^{+}$rates in CD8 and CD4 T cells. On the basis of the positive rates of PD-1 in the two types of intratumoral $\mathrm{T}$ cells, cancer patients were divided into high $\mathrm{PD}-1^{+}-\mathrm{CD} 8$ ( $>$ median expression rate: $27.8 \%$ ) and low PD-1 ${ }^{+}$-CD8 (<median expression rate: $27.8 \%$ ) groups, or into high $\mathrm{PD}-1^{+}-\mathrm{CD} 4$ ( $>$ median expression rate: $14.5 \%$ ) and low $\mathrm{PD}-1^{+}$CD4 (<median expression rate: $14.5 \%$ ) groups. Alternatively, based on the cell types expressing B7-H1 in tumor tissue, the cancer patients were grouped into $\mathrm{B} 7-\mathrm{H} 1^{+}$in $\mathrm{EpCAM}^{+}$tumor cells vs $\mathrm{B}-\mathrm{H} 1^{+}$in $\mathrm{EpCAM}^{-}$stromal cells. Student's $t$-test was also used to examine whether the different expression patterns of PD-1 or B7-H1 were associated with age. $\mathrm{A} \chi^{2}$ test was used to assess whether expression patterns of PD-1 or B7-H1 correlated with other clinicopathological parameters such as gender and clinical tumor-node-metastasis (TNM) staging. Overall survival, disease-free survival, locoregional relapse-free survival, and distant metastasis-free survival were measured from the initial diagnosis until death or specific events; follow-up data of surviving patients were assessed on the last contact date. Kaplan-Meier analysis was performed to estimate the survival distribution, and a log-rank test was used to evaluate the correlation between expression patterns of PD-1 or B7-H1 with the posttreatment survival. Multivariate Cox proportional hazard regression modeling was used to test the influence of covariates (PD-1 + $-\mathrm{CD} 8$ rate, $\mathrm{PD}-1^{+}$-CD4 rate, gender, age, histological type, tumor stage, nodal status, and clinical staging) on locoregional relapse-free, disease-free, and overall survival. $P$-values are two sided. All analyses were performed using SPSS 13.0 statistical software (SPSS, Chicago, IL, USA).

\section{Results}

\section{PD-1 Expression in CD8 and CD4 Cells in Nasopharyngeal Carcinoma and Control Tissue}

As both CD8 and CD4 cells can be involved in controlling tumor growth, we used immunofluorescence co-staining to simultaneously detect the expression of PD-1 as well as CD8 and CD4 in paraffined tissue sections. Substantially different patterns of PD-1 were detected in nasopharyngeal tumor and control tissue (Figure 1). PD-1 was costained with CD4, but not CD8 cells in the control specimens diagnosed as lymphoid hyperplasia (Figures 1a and b). A similar staining pattern was also reported in tonsils, ${ }^{16}$ which suggests that PD-1 is expressed predominantly by CD4 but not CD8 cells during an active immune response in lymphoid tissue. In contrast, PD-1 was markedly detected in not only CD4, but also CD8 cells in tumor tissue (Figures 1a and b), which indicated preferential increase of PD-1 expression in the intratumoral CD8 cells. The $\mathrm{PD}-1^{+}$percentage of CD8 cells was significantly higher in tumor tissue than in control tissue (mean: 28.4 vs 3.9\%, 95\% confidence interval (CI): 23.93-32.79\% vs 1.57$6.25 \%, P<0.0001$; Figure 1c); however, PD-1 expression rates in CD4 cells were not significantly different between tumor and control tissue (mean: 15.6 vs $20.5 \%$, 95\% CI: $12.91-18.20 \%$ vs $14.56-$ $26.35 \%$; Figure 1d). The PD-1 expression rates of CD8 and CD4 cells were not associated (data not shown). 
a
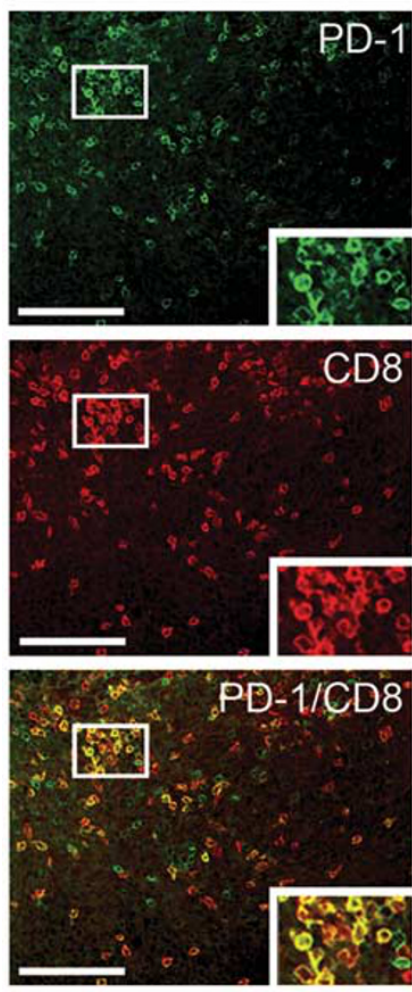

Controls
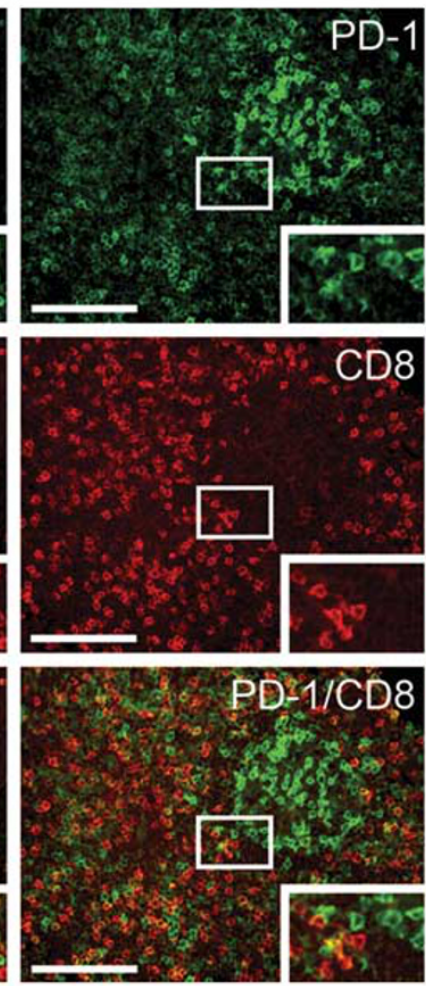

b
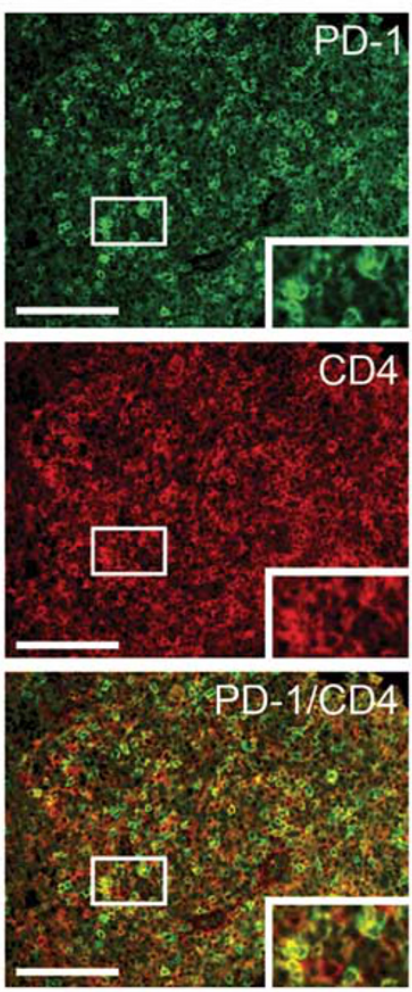
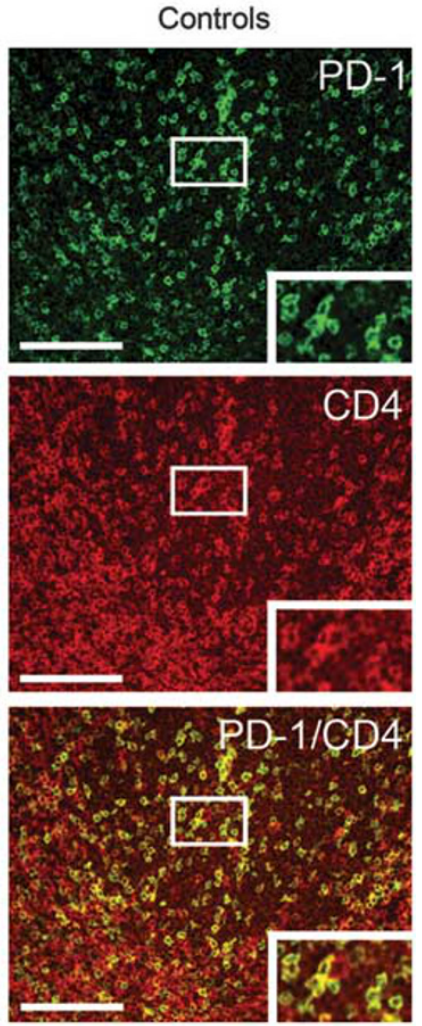
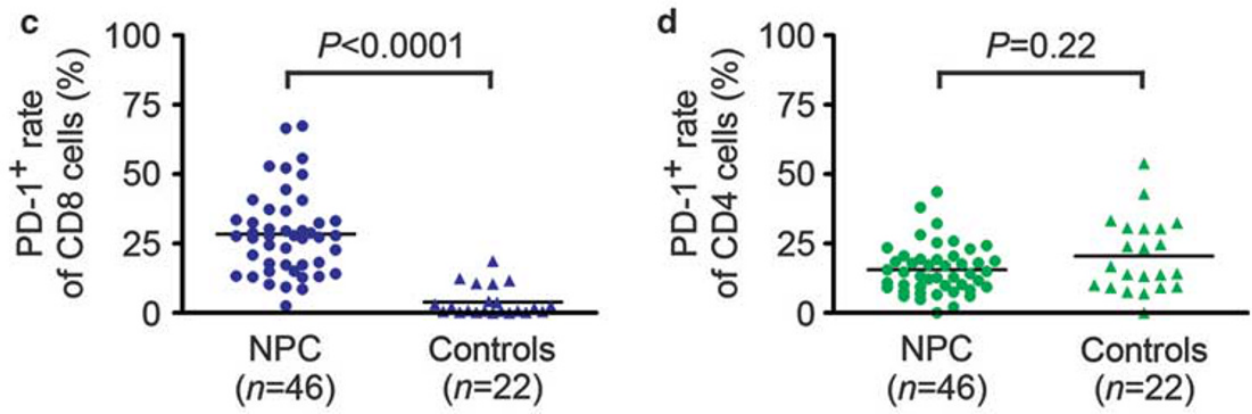

Figure 1 PD-1 expression in CD4 and CD8 T cells in nasopharyngeal carcinoma tumor (NPC) and control nasopharyngeal tissue. (a) Representative results of immunofluorescence staining of PD-1 and CD8 in NPC and control tissue. (b) Representative results of immunofluorescence staining of PD-1 and CD4 in NPC and control tissue. PD-1-positive (PD-1 ${ }^{+}$) cells are stained green; CD8 and CD4 cells are stained red. PD-1 ${ }^{+}$-CD8 and PD-1+ ${ }^{+}$CD4 cells in the merged images (third row) are yellow. Scale bar, $100 \mu \mathrm{m}$. (c) PD-1 ${ }^{+}$-CD8 rates in NPC and control tissue. (d) PD-1 $1^{+}$-CD4 rates in NPC and control tissue. The expression rates in 46 NPC and 22 control samples are plotted; each dot represents one specimen. Black bars $=$ mean values.

\section{Association of PD-1 Expression in Intratumoral CD4 and CD8 Cells with Clinicopathological Parameters and Post-treatment Outcome of Nasopharyngeal Carcinoma}

To analyze whether PD-1 expression correlates with clinicopathological parameters or the prognosis of nasopharyngeal carcinoma, we divided the cancer patients according to their PD-1-positive percentage of intratumoral CD8 or CD4 cells: high PD- $1^{+}$-CD8 group (>median expression rate: $27.8 \% ; 23$ cases) vs low PD-1 ${ }^{+}-\mathrm{CD} 8$ group ( $<$ median expression rate: $27.8 \% ; 23$ cases), and high $\mathrm{PD}-1^{+}-\mathrm{CD} 4$ group ( $>$ median expression rate: $14.5 \% ; 23$ cases) vs low
PD-1 ${ }^{+}$-CD4 group $(<$median expression rate: $14.5 \%$; 23 cases). PD-1 expression rates and the clinicopathological parameters of age, gender, tumor stage, nodal involvement, and clinical TNM staging at diagnosis were not correlated (Table 1). Notably, a Kaplan-Meier survival analysis showed that the patients in the high PD-1+-CD8 group had poorer post-treatment outcomes than did the patients in the low PD- $1^{+}-\mathrm{CD} 8$ group. The prognoses for overall survival $(P=0.05$; Figure 2a) and disease-free survival $(P=0.007$; Figure $2 \mathrm{~b})$ were significantly worse in the high PD-1+-CD8 group. An analysis of the types of treatment failure showed that a high PD-1 $1^{+}-\mathrm{CD} 8$ rate strongly correlated with a locoregional relapse 
Table 1 Correlation between clinicopathological parameters and expression of PD-1 or B7-H1 in biopsies of nasopharyngeal carcinoma

\begin{tabular}{|c|c|c|c|c|c|c|c|c|c|}
\hline \multirow[t]{2}{*}{ Parameter } & \multicolumn{3}{|c|}{ PD-1 expression rate of CD8 TILs } & \multicolumn{3}{|c|}{ PD-1 expression rate of CD4 TILs } & \multicolumn{3}{|c|}{ B7-H1 expression } \\
\hline & $\begin{array}{c}\text { Low PD-1-CD8 } \\
\quad(<27.8 \%) \\
(\mathrm{n}=23)\end{array}$ & $\begin{array}{l}\text { High PD-1-CD8 } \\
\quad(>27.8 \%) \\
(\mathrm{n}=23)\end{array}$ & P-value & $\begin{array}{c}\text { Low } P D-1-C D 4 \\
(<14.5 \%) \\
(\mathrm{n}=23)\end{array}$ & $\begin{array}{c}\text { High PD-1-CD4 } \\
\quad(>14.5 \%) \\
(\mathrm{n}=23)\end{array}$ & $\mathrm{P}$-value & $\begin{array}{c}B 7-H 1^{+} \text {in } \\
\text { EpCAM cells } \\
(\mathrm{n}=10)\end{array}$ & $\begin{array}{c}B 7-H 1^{+} \text {in } \\
\text { EpCAM cells } \\
(\mathrm{n}=18)\end{array}$ & P-value \\
\hline Age (mean, 95\% CI) & $50.8,46.7-54.9$ & $49.7,44.0-55.5$ & $0.76^{\mathrm{a}}$ & $51.7,47.0-56.3$ & $48.9,43.7-54.1$ & $0.42^{\mathrm{a}}$ & $50.9,40.6-61.2$ & $52.3,46.5-58.1$ & $0.78^{\mathrm{a}}$ \\
\hline \multicolumn{10}{|l|}{ Gender } \\
\hline Male & $19(82.6 \%)$ & $20(87.0 \%)$ & $0.68^{\mathrm{b}}$ & $21(91.3 \%)$ & $18(78.3 \%)$ & $0.22^{\mathrm{b}}$ & $7(70.0 \%)$ & $11(61.1 \%)$ & $0.70^{\mathrm{b}}$ \\
\hline Female & $4(17.4 \%)$ & $3(13.0 \%)$ & & $2(8.7 \%)$ & $5(21.7 \%)$ & & $3(30.0 \%)$ & $7(38.9 \%)$ & \\
\hline \multicolumn{10}{|l|}{ Tumor stage } \\
\hline T1 & $6(26.1 \%)$ & $5(21.7 \%)$ & $0.60^{\mathrm{b}}$ & $6(26.1 \%)$ & $5(21.7 \%)$ & $0.81^{\mathrm{b}}$ & $4(40.0 \%)$ & $6(33.3 \%)$ & $0.72^{\mathrm{b}}$ \\
\hline $\mathrm{T} 2$ & $6(26.1 \%)$ & $7(30.4 \%)$ & & $5(21.7 \%)$ & $8(34.8 \%)$ & & $1(10.0 \%)$ & $2(11.1 \%)$ & \\
\hline T3 & $5(21.7 \%)$ & $8(34.8 \%)$ & & $8(34.8 \%)$ & $5(21.7 \%)$ & & $4(40.0 \%)$ & $5(27.8 \%)$ & \\
\hline $\mathrm{T} 4$ & $6(26.1 \%)$ & $3(13.0 \%)$ & & $4(17.4 \%)$ & $5(21.7 \%)$ & & $1(10.0 \%)$ & $5(27.8 \%)$ & \\
\hline \multicolumn{10}{|l|}{ Nodal status } \\
\hline No & $3(13.0 \%)$ & $2(8.7 \%)$ & $0.89^{b}$ & $3(13.0 \%)$ & $2(8.7 \%)$ & $0.64^{\mathrm{b}}$ & $3(30.0 \%)$ & $4(22.2 \%)$ & $0.59^{\mathrm{b}}$ \\
\hline N1 & $6(26.1 \%)$ & $6(26.1 \%)$ & & $7(30.4 \%)$ & $5(21.7 \%)$ & & $4(40.0 \%)$ & $6(33.3 \%)$ & \\
\hline N2 & $8(34.8 \%)$ & $10(43.5 \%)$ & & $7(30.4 \%)$ & $11(47.8 \%)$ & & $3(30.0 \%)$ & $5(27.8 \%)$ & \\
\hline N3 & $6(26.1 \%)$ & $5(21.7 \%)$ & & $6(26.1 \%)$ & $5(21.7 \%)$ & & $0(0 \%)$ & $3(16.7 \%)$ & \\
\hline \multicolumn{10}{|l|}{ TNM staging } \\
\hline 1 & $1(4.4 \%)$ & $0(0 \%)$ & $0.36^{\mathrm{b}}$ & $1(4.4 \%)$ & $0(0 \%)$ & $0.85^{\mathrm{b}}$ & $1(10.0 \%)$ & $2(11.1 \%)$ & $0.17^{\mathrm{b}}$ \\
\hline 2 & $4(17.4 \%)$ & $4(17.4 \%)$ & & $5(21.7 \%)$ & $3(13.0 \%)$ & & $3(30.0 \%)$ & $3(16.7 \%)$ & \\
\hline 3 & $7(30.4 \%)$ & $12(52.2 \%)$ & & $9(39.1 \%)$ & $10(43.5 \%)$ & & $5(50.0 \%)$ & $4(22.2 \%)$ & \\
\hline 4 & $11(47.8 \%)$ & $7(30.4 \%)$ & & $8(34.8 \%)$ & $10(43.5 \%)$ & & $1(10.0 \%)$ & $9(50.0 \%)$ & \\
\hline
\end{tabular}

${ }^{\text {a }} P$-values were obtained from Student's $t$-test.

${ }^{\mathrm{b}} P$-values were obtained from $\chi^{2}$ tests. 


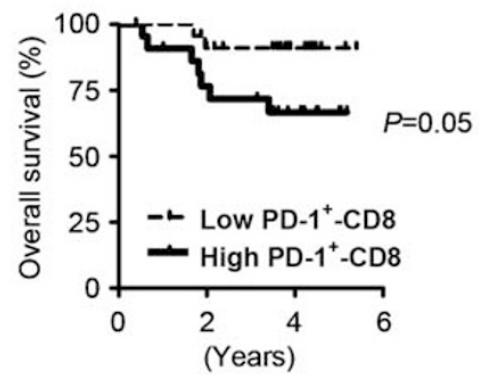

C

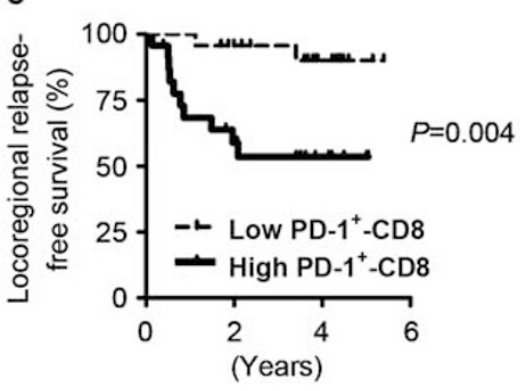

e

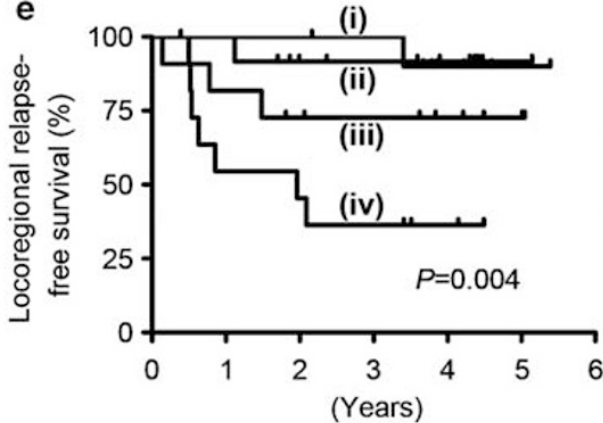

b

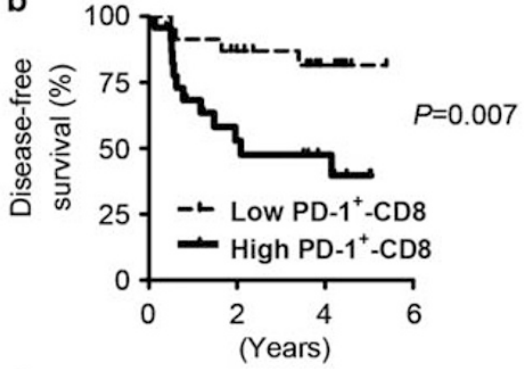

d

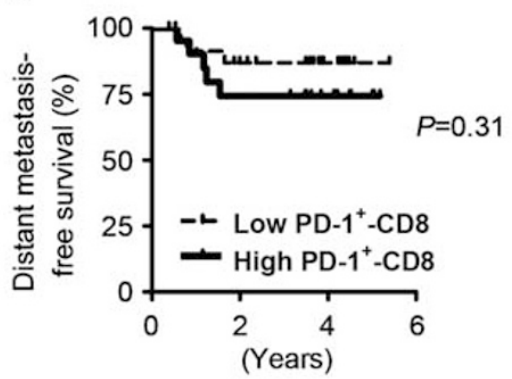

(i) $<16 \% \mathrm{PD}-1^{+}-\mathrm{CD} 8$

(ii) $16 \%-27.8 \% \mathrm{PD}-1^{+}-\mathrm{CD} 8$

(iii) $27.8 \%-35 \%$ PD-1 ${ }^{+}$-CD8

(iv) $>35 \%$ PD- ${ }^{+}$-CD 8
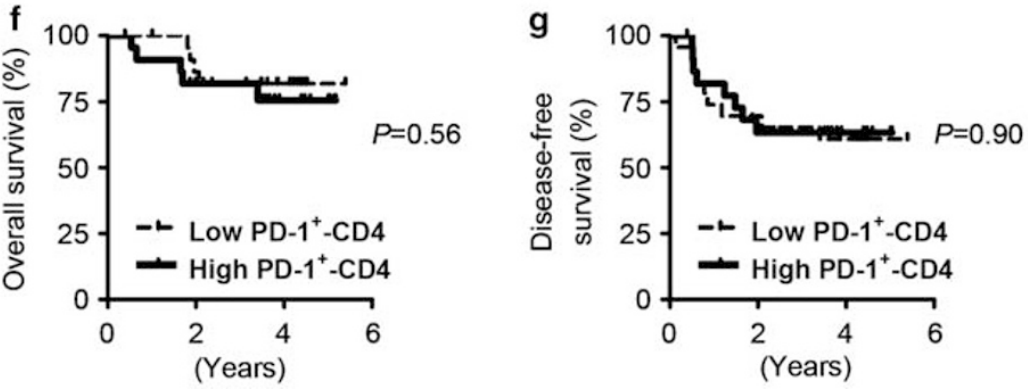

Figure 2 Kaplan-Meier analysis of the correlation between PD-1 expression in intratumoral CD4 and CD8 cells and post-treatment survival of nasopharyngeal carcinoma patients. (a) Correlation between PD- $1^{+}$-CD8 expression and overall survival. (b) Correlation between PD-1+ $1^{+}$CD8 expression and disease-free survival. (c) Correlation between PD-1 $1^{+}$-CD8 expression and locoregional recurrencefree survival. (d) Correlation between PD-1 ${ }^{+}-\mathrm{CD} 8$ expression and distant metastasis-free survival. (e) Correlation between PD-1 ${ }^{+}-\mathrm{CD} 8$ expression and locoregional recurrence-free survival (four groups). (f) Correlation between PD-1 ${ }^{+}$-CD4 expression and overall survival. (g) Correlation between PD-1+-CD4 expression and disease-free survival.

of nasopharyngeal carcinoma ( $P=0.004$; Figure 2c). Although the high PD-1+ ${ }^{+}$CD8 group also had more distant metastasis than did the low $\mathrm{PD}-1^{+}-\mathrm{CD} 8$ group, the difference was not significant (Figure 2d). We also stratified the cancer patients into four groups based on their PD-1+CD8 percentage, and still observed a pronounced trend that the incidence of locoregional recurrence increased as the PD-1 expression rate of CD8 cells rose; the group with the highest $\mathrm{PD}-1^{+}$rate showed the highest incidence of recurrence $\left(P=0.004\right.$; Figure 2e). However, the PD- $1^{+}$ -CD4 expression rate was not correlated with the overall or disease-free survival of the cancer patients (Figures 2f and g). In both univariate and multivariate analyses, a high $\mathrm{PD}-1^{+}-\mathrm{CD} 8$ rate, but not a high PD-1 ${ }^{+}-\mathrm{CD} 4$ rate, significantly predicted a poor prognosis after therapy, even though the TNM staging did not reliably predict clinical outcome in this study (Tables 2 and 3). Multivariate analysis showed that, compared with the low $\mathrm{PD}-1^{+}-\mathrm{CD} 8$ 
Table 2 Univariate analysis to assess the association of clinicopathological parameters with prognosis of nasopharyngeal carcinoma

\begin{tabular}{|c|c|c|c|c|}
\hline \multirow[t]{2}{*}{ Parameter } & \multirow{2}{*}{$\begin{array}{l}\text { Prognostic factor } \\
\text { Case number/total, percentage }\end{array}$} & \multicolumn{3}{|c|}{ Univariate analysis $\left(\mathrm{P}-\right.$-value $\left.^{\mathrm{a}}\right)$} \\
\hline & & $L R F S$ & $D F S$ & OS \\
\hline $\mathrm{PD}-1^{+}$rate of CD8 $\mathrm{T}$ cells & $\begin{array}{l}\text { High PD-1-CD8 (>27.8\%) } \\
23 / 46,50 \%\end{array}$ & 0.004 & 0.007 & 0.050 \\
\hline $\mathrm{PD}-1^{+}$rate of CD4 $\mathrm{T}$ cells & $\begin{array}{l}\text { High PD-1-CD4 (>14.5\%) } \\
23 / 46,50 \%\end{array}$ & 0.989 & 0.900 & 0.560 \\
\hline Gender & $\begin{array}{l}\text { Male (vs female) } \\
39 / 46,84.8 \%\end{array}$ & 0.946 & 0.653 & 0.933 \\
\hline Age & $\begin{array}{l}>50 \text { years } \\
19 / 46,41.3 \%\end{array}$ & 0.334 & 0.883 & 0.758 \\
\hline Histological type & $\begin{array}{l}\text { WHO type } 3 \text { (vs WHO type } 2 \text { ) } \\
35 / 46,76.1 \%\end{array}$ & 0.726 & 0.502 & 0.348 \\
\hline Tumor stage & $\begin{array}{l}\text { T3/4 (vs T1/2) } \\
22 / 46,47.8 \%\end{array}$ & 0.474 & 0.351 & 0.546 \\
\hline Nodal status & $\begin{array}{l}\mathrm{N} 2 / 3(\text { vs No/1) } \\
29 / 46,63.0 \%\end{array}$ & 0.671 & 0.939 & 0.951 \\
\hline TNM staging & $\begin{array}{l}\text { Stage } 3 / 4 \text { (vs stage } 1 / 2) \\
37 / 46,80.4 \%\end{array}$ & 0.86 & 0.314 & 0.531 \\
\hline
\end{tabular}

LRFS, locoregional relapse-free survival; DFS, disease-free survival; OS, overall survival.

${ }^{\text {a }} P$-values were obtained from log-rank test. Bold values signify $P$-value $\leq 0.05$.

group, the high $\mathrm{PD}-1^{+}-\mathrm{CD} 8$ group had a 6.5 times higher risk of locoregional recurrence (95\% CI: 1.7724.01, $P=0.005$ ), a 6.5 times higher risk of treatment failure (95\% CI: $1.48-28.22, P=0.013$ ), and a 9.5 times higher risk of death (95\% CI: 1.55-58.26, $P=0.015$ ), which suggested that PD-1 expression in intratumoral CD8 cells may have an independent effect on the post-treatment outcome of nasopharyngeal carcinoma.

\section{PD-1 Ligand Expression in Nasopharyngeal Carcinoma and Control Tissue}

Other studies have reported that the expression of PD-1 ligands, especially of B7-H1, is higher in tumor tissue than in control tissue, and that it is associated with a poor prognosis. ${ }^{20-23}$ Therefore, we also used immunofluorescence co-staining of individual PD-1 ligands (B7-H1 and B7-DC) and an epithelial marker EpCAM to check the cell types expressing the ligands in the tumor and control tissue. As staining antibodies for these markers were only suitable for frozen sections, we used tissue sections of snapfrozen specimens from a separate group of patients. B7-DC was not detected in any of the specimens (data not shown), but B7-H1 was positive in 28 $(100 \%)$ tumor samples and in 23 of $29(79 \%)$ controls. Both the tumor and control samples showed two distinct staining patterns of B7-H1 (Figures 3a and b). In 18 of 28 (64\%) tumor specimens, B7-H1 was detected predominantly in EpCAM $^{+}$epithelial cells, but in the remaining 10, B7-H1 was detected exclusively in EpCAM ${ }^{-}$stromal cells (Figures 3a and 4a). Of the $23 \mathrm{~B} 7-\mathrm{H}^{+}{ }^{+}$control samples, 8 cases showed B7-H1 staining in EpCAM ${ }^{+}$ epithelial cells and 15 in EpCAM $^{-}$cells (Figures 3b and $4 \mathrm{a})$.

\section{Association of B7-H1 Expression with Clinicopathological Parameters and Post-treatment Outcome of Nasopharyngeal Carcinoma}

As B7-H1 was expressed at similar levels in all nasopharyngeal carcinoma biopsy samples, we divided the cancer patients into two groups: B7-H1 ${ }^{+}$ in $\mathrm{EpCAM}^{+}$epithelial cells vs $\mathrm{B} 7-\mathrm{H}^{+}{ }^{+}$in $\mathrm{EpCAM}^{-}$ stromal cells. The clinicopathological parameters of age, gender, tumor stage, nodal status, and TNM staging were not significantly different between the groups (Table 1), nor were there significant differences in post-treatment overall survival or diseasefree survival (Figures $4 \mathrm{~b}$ and c).

\section{Discussion}

In summary, we found that the expression of PD- 1 in CD8 $\mathrm{T}$ cells was significantly higher in nasopharyngeal tumor tissue than in non-cancerous nasopharyngeal tissue. In addition, we found that the high expression rate of PD-1 in intratumoral CD8 cells predicted a poor clinical outcome in terms of mortality, treatment failure, and locoregional recurrence. However, the expression of PD-1 in CD4 $\mathrm{T}$ cells and of B7-H1 in epithelial and stromal cells was not significantly different between tumor and non-cancerous control tissue, and its expression was not associated with clinical outcome of the cancer patients.

During a chronic viral infection, persistent stimulation with viral antigens exhausts virus-specific T cells: they are rendered phenotypically activated, but functionally impaired. ${ }^{7,8}$ PD-1 has been recognized as a remarkable marker for the exhausted T cells. ${ }^{7,9,10,12}$ Consistent with the idea that continuous exposure to tumor antigens may also facilitate the exhaustion of infiltrating lymphocytes in tumor 


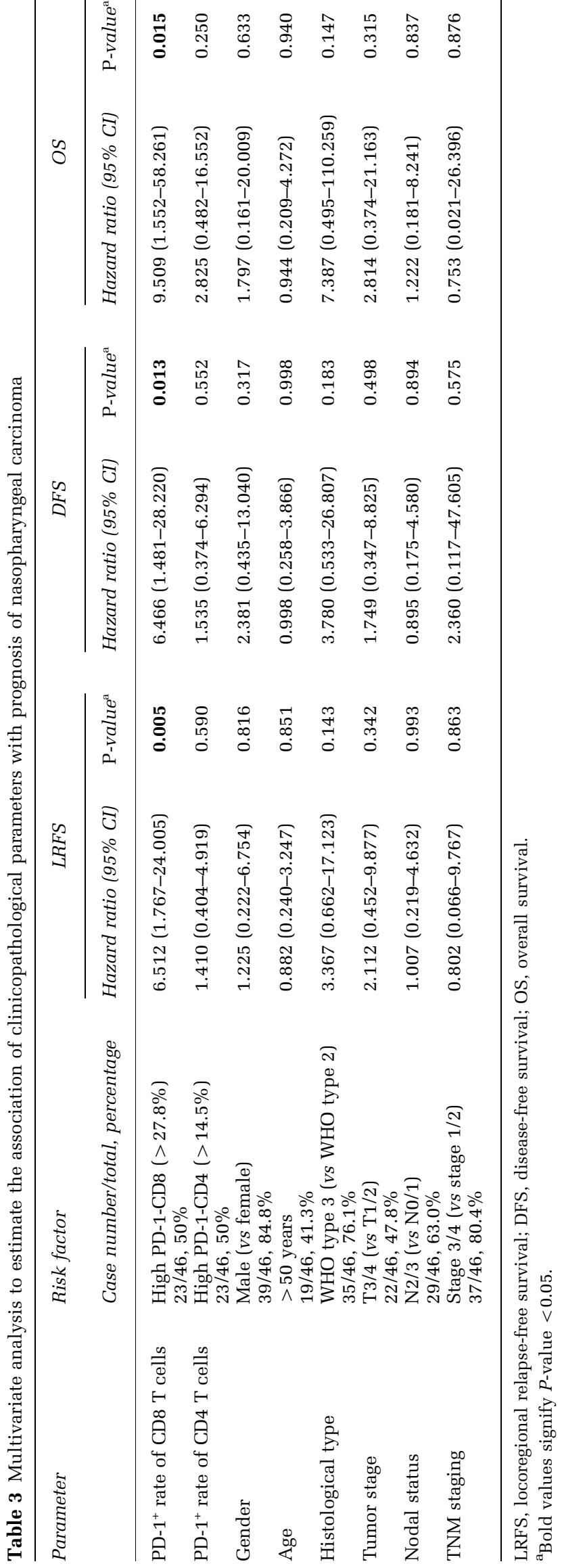

tissue, recent studies have reported an association between PD-1 expression and the functional impairment of TILs in some cancers. ${ }^{13-15}$ Nasopharyngeal carcinoma tumors, especially those of WHO type 2 and type 3 in the endemic areas, are characterized by a prevailing Epstein-Barr virus infection in cancerous cells with intensive lymphocytic infiltration and, therefore, represent a tissue microenvironment for both tumor development and chronic viral infection. ${ }^{1} \mathrm{PD}-1$ expression in the TILs suggests that T-cell exhaustion may also occur in this cancer, which is supported by previous findings that the cytotoxic activity and cytokine production of T lymphocytes in nasopharyngeal carcinoma tumors are defective even if some of the TILs exhibit activated phenotypes. ${ }^{24,25}$ Additional studies are required to determine whether the $\mathrm{PD}$-1-expressing TILs are specific to antigens of the tumor cells or of Epstein-Barr virus, and whether they are functionally exhausted.

Impairment of anti-tumor immune responses prevents the body from clearing residual tumor cells after radiotherapy or chemotherapy, which increases the risk of tumor recurrence after treatment. ${ }^{4,5}$ Multiple immunosuppressive mechanisms in nasopharyngeal carcinoma have been proposed: interleukin-10, ${ }^{26}$ transforming growth factor $\beta 1,{ }^{27}$ Fas ligand ${ }^{28}$ regulatory $\mathrm{T}$ cells, ${ }^{29}$ and galectin- $9 .{ }^{30}$ As most of these mechanisms converge to inhibit effector $\mathrm{T}$ cells, we hypothesized that a marker summarizing the suppressive status of TILs would help identify nasopharyngeal cancer patients with a high risk of treatment failure after standard radiotherapy or chemotherapy. PD-1 is a candidate marker because it is not only a specific receptor that suppresses T cells, but it is also an indicator of the severity of T-cell dysfunction. ${ }^{31,32}$ PD-1 expression in primary nasopharyngeal carcinoma tumors was higher in CD8 T cells, the major cytotoxic effector cells that eliminate tumor cells, than in CD4 T cells. This finding suggests that CD8 cells are selectively exhausted in the tumor microenvironment, which may explain the significant association of the high $\mathrm{PD}-1^{+}-\mathrm{CD} 8$ group with frequent locoregional recurrence. However, its weak association with distant metastasis implies that the local immunosuppression status at primary tumor sites is not necessarily linked to the status at metastatic sites, but we did not use metastatic tumor tissue to test this hypothesis.

Our finding of a preferential increase of $\mathrm{PD}-1^{+}$ CD8 cells in nasopharyngeal carcinoma tumors differs from the findings of studies that reported elevated PD-1 expression in both CD4 and CD8 cells in melanoma, Hodgkin lymphoma, and cervical carcinoma tissue. ${ }^{13,15,33}$ The high $\mathrm{PD}-1^{+}$-CD8 rate was significantly correlated with a poor prognosis in our study. In contrast, for follicular lymphoma, in which PD-1 is highly expressed by CD4 cells, higher PD-1 expression in TILs predicts improved overall survival. ${ }^{16}$ We hypothesize that PD-1 expression in 
a $\mathrm{NPC}(\mathrm{I})$
$\mathrm{B}^{-}-\mathrm{H} 1^{+}$in
$\mathrm{EpCAM}^{+}$cells
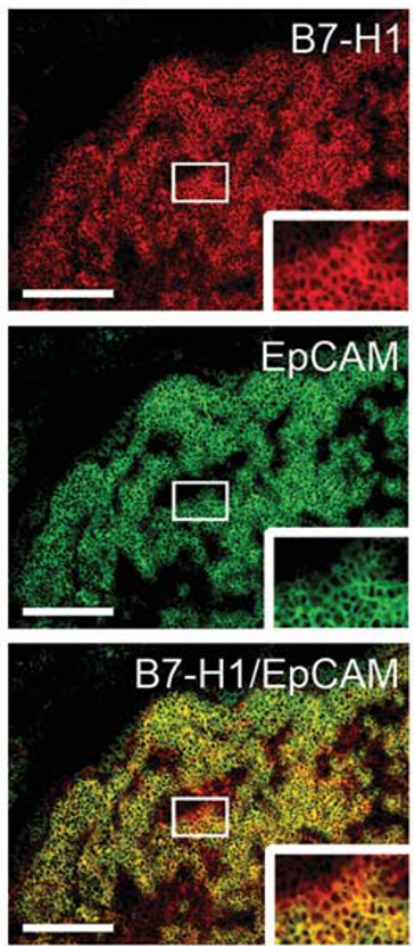

NPC (II)

$\mathrm{B} 7-\mathrm{H}^{+}{ }^{+}$in

$\mathrm{EpCAM}^{-}$cells
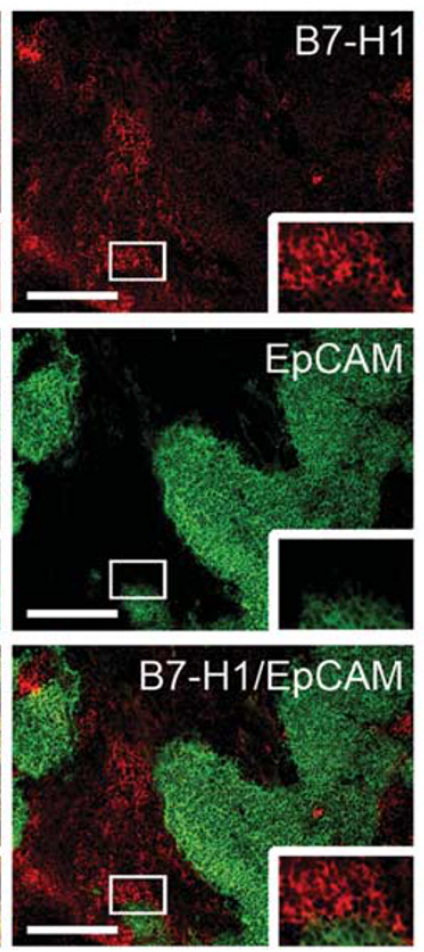

b

Control (I)

$\mathrm{B} 7-\mathrm{H}^{+}{ }^{+}$in

$\mathrm{EpCAM}^{+}$cells
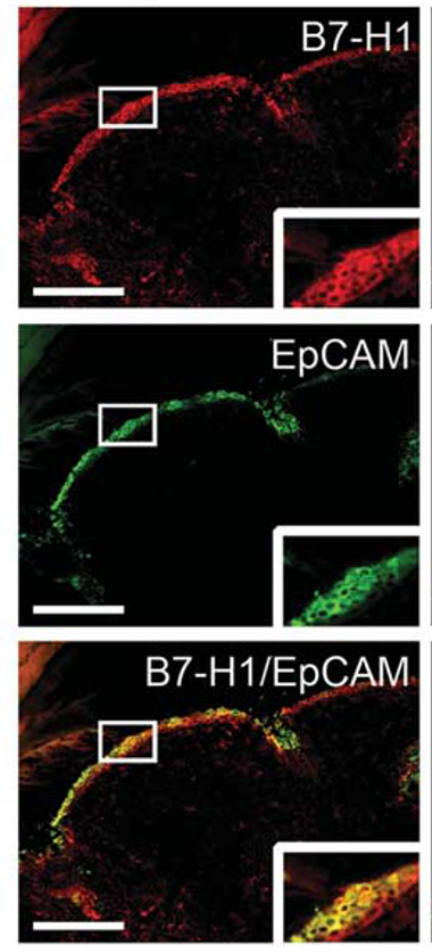

Control (II)

$$
\mathrm{B} 7-\mathrm{H} 1^{+} \text {in }
$$

EpCAM $^{-}$cells
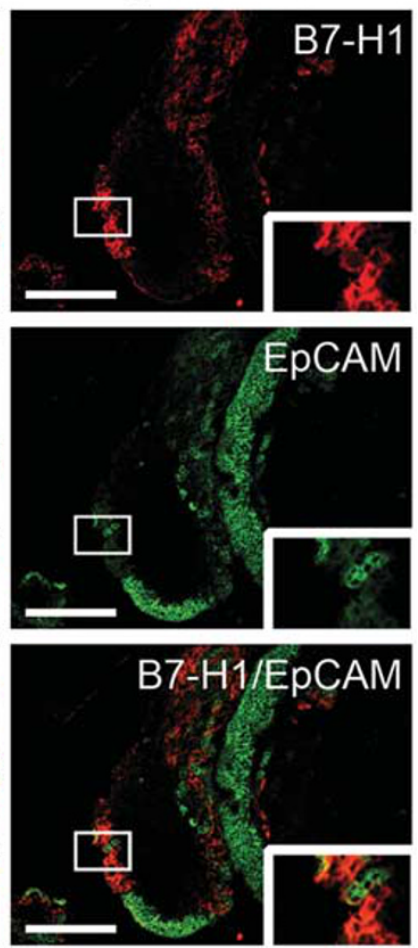

Figure 3 Immunofluorescence detection of B7-H1 and EpCAM in nasopharyngeal carcinoma tumor (NPC) and control tissue. (a) Representative staining patterns of B7-H1 and EpCAM in two tumor samples. (b) Representative staining patterns of B7-H1 and EpCAM in two control samples. B7-H1 ${ }^{+}$staining is red; $\mathrm{EpCAM}^{+}$staining is green. $\mathrm{B} 7-\mathrm{H} 1^{+}$and $\mathrm{EpCAM}^{+}$co-staining in the merged images (third row) is yellow. Scale bar, $200 \mu \mathrm{m}$.

different types of intratumoral lymphocytes is likely to differentially influence clinical outcome, which may account for the variable prognostic impact of PD-1 in studies that did not discriminate between the types of PD-1+ TILs. ${ }^{16-19}$ Therefore, to evaluate the clinical relevance of PD-1, the types of PD-1expressing cells should be taken into consideration. Interestingly, a poor prognosis for nasopharyngeal carcinoma has been predicted by a large number of CD8 cells expressing granzyme $\mathrm{B}$, a marker of activated cytotoxic $\mathrm{T}$ cells. ${ }^{34}$ It remains to be clarified whether those granzyme B-positive TILs express PD-1 and are functionally suppressed.

Our findings are different from the previous reports that $\mathrm{B} 7-\mathrm{H} 1$ expression is upregulated in many kinds of tumors and predicts a poor clinical outcome. ${ }^{20-23}$ Although we were unable to obtain both parafinned and frozen specimens from the same patients and examine PD-1 and its ligands in the same tissue samples, we found that B7-H1 was expressed generally in nasopharyngeal carcinoma and control tissue, and either EpCAM ${ }^{+}$epithelial tumor cells or EpCAM ${ }^{-}$stromal cells are the source of this PD-1 ligand. B7-H1 expression in the noncancerous control tissues is consistent with a previous report that $\mathrm{B} 7-\mathrm{H} 1$ is induced in infected or inflamed nasal tissue. ${ }^{35}$ Thus, unlike in many other cancers, B7-H1 expression is not specific to malignant tissue in the nasopharynx. Abundant B7$\mathrm{H} 1$ expression in all the tested tumor biopsy tissue samples suggests that this ligand is probably not a limiting factor for PD-1-mediated suppression of TILs in nasopharyngeal carcinoma. Our finding that B7-H1 was not associated with the clinical prognosis of the cancer supports this notion. Therefore, it is possible that in nasopharyngeal carcinoma tumors, the cells that express PD-1, rather than those that express its ligands, are critical for determining the extent of TIL dysfunction and for affecting the clinical outcome.

We conclude that PD- ${ }^{+}$-CD8 cells are a prognostic marker of nasopharyngeal carcinoma. Our findings have two major clinical implications. First, because cancer patients with low $\mathrm{PD}-1^{+}-\mathrm{CD} 8$ expression respond well to conventional therapies, efforts to reduce the damage and side effects of standard treatments should benefit them the most. Second, patients with high PD-1 ${ }^{+}$-CD8 expression should be carefully monitored after treatments and considered for advanced therapies. ${ }^{2}$ The inhibitory effects of PD-1 and B7-H1 can be overcome by certain immunoactivators, ${ }^{36,37}$ and blocking PD-1 
a

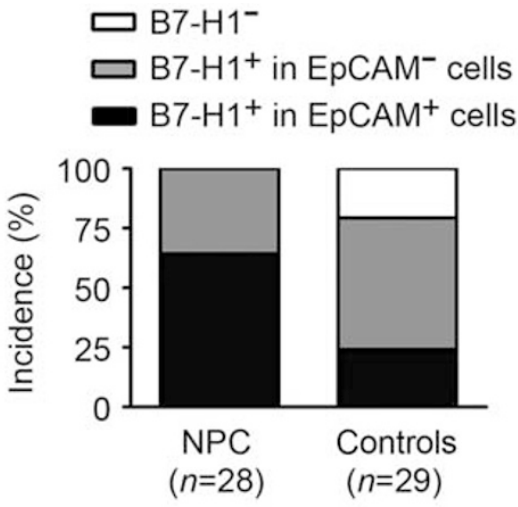

b
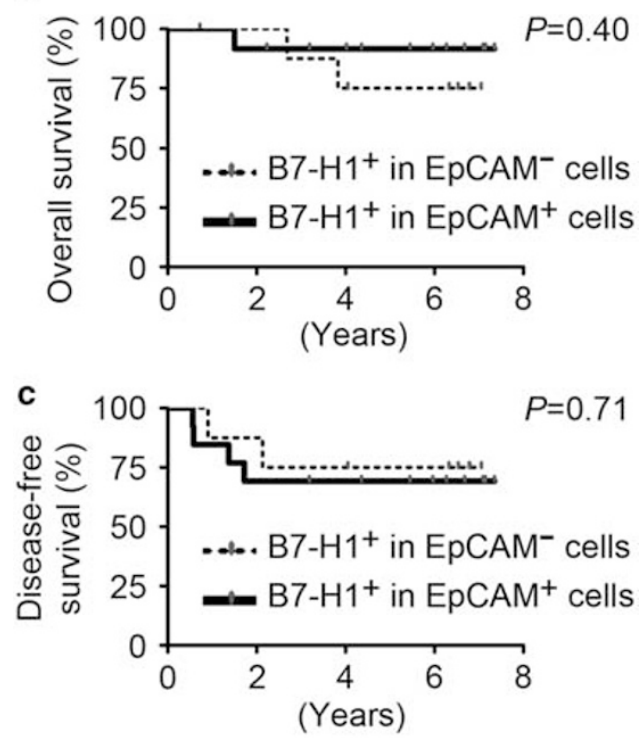

Figure 4 B7-H1 expression in nasopharyngeal carcinoma tumor (NPC) and control tissue, and its clinical relevance to posttreatment survival of cancer patients. (a) Distribution of the B7-H1 staining patterns in 28 NPC and 29 control tissue samples. (b) Correlation between B7-H1 staining patterns and overall survival. (c) Correlation between B7-H1 staining patterns and disease-free survival.

and B7-H1 restores the anti-tumor activity of TILs in some cancers, ${ }^{14,15,22}$ which indicates that local immunosuppression in tumors can be reversed. Therefore, we hypothesize that alleviating the strong local immunosuppression of nasopharyngeal carcinoma by counteracting PD-1 and its ligands will not only improve the outcomes of Epstein-Barr virustargeted cancer immunotherapy, but also improve the efficacy of conventional nasopharyngeal carcinoma therapy.

\section{Acknowledgements}

We thank Fan-Chen Tseng and Jeffrey Chang for advice about statistical analysis, and Chaio-Wei Chen and Mei-Ling Tsai for technical assistance. This study was supported by the Taiwan National Health Research Institutes (grant numbers CL-097-
PP-15, CL-098-PP-15, and ID-099-PP-16) and the Comprehensive Cancer Center in Southern Taiwan (DOH99-TD-C-111-003).

\section{Disclosure/conflict of interest}

The authors declare no conflict of interest.

\section{References}

1 Spano JP, Busson P, Atlan D, et al. Nasopharyngeal carcinomas: an update. Eur J Cancer 2003;39: 2121-2135.

$2 \mathrm{Ma} \mathrm{BB}$, Hui EP, Chan AT. Systemic approach to improving treatment outcome in nasopharyngeal carcinoma: current and future directions. Cancer Sci 2008;99:1311-1318.

3 Chan AT, Teo PM, Johnson PJ. Nasopharyngeal carcinoma. Ann Oncol 2002;13:1007-1015.

4 Apetoh L, Ghiringhelli F, Tesniere A, et al. Toll-like receptor 4-dependent contribution of the immune system to anticancer chemotherapy and radiotherapy. Nat Med 2007;13:1050-1059.

5 Lake RA, van der Most RG. A better way for a cancer cell to die. N Engl J Med 2006;354:2503-2504.

6 Stewart TJ, Abrams SI. How tumours escape mass destruction. Oncogene 2008;27:5894-5903.

7 Keir ME, Butte MJ, Freeman GJ, et al. PD-1 and its ligands in tolerance and immunity. Annu Rev Immunol 2008;26:677-704.

8 Sharpe AH, Wherry EJ, Ahmed R, et al. The function of programmed cell death 1 and its ligands in regulating autoimmunity and infection. Nat Immunol 2007;8: 239-245.

9 Barber DL, Wherry EJ, Masopust D, et al. Restoring function in exhausted CD8 T cells during chronic viral infection. Nature 2006;439:682-687.

10 Day CL, Kaufmann DE, Kiepiela P, et al. PD-1 expression on HIV-specific T cells is associated with T-cell exhaustion and disease progression. Nature 2006;443:350-354.

11 Urbani S, Amadei B, Tola D, et al. PD-1 expression in acute hepatitis $\mathrm{C}$ virus (HCV) infection is associated with HCV-specific CD8 exhaustion. J Virol 2006; 80:11398-11403.

12 Trautmann L, Janbazian L, Chomont N, et al. Upregulation of PD-1 expression on HIV-specific CD8+ T cells leads to reversible immune dysfunction. Nat Med 2006;12:1198-1202.

13 Ahmadzadeh M, Johnson LA, Heemskerk B, et al. Tumor antigen-specific CD8 $\mathrm{T}$ cells infiltrating the tumor express high levels of PD-1 and are functionally impaired. Blood 2009;114:1537-1544.

14 Wu K, Kryczek I, Chen L, et al. Kupffer cell suppression of CD8+ $\mathrm{T}$ cells in human hepatocellular carcinoma is mediated by B7-H1/programmed death1 interactions. Cancer Res 2009;69:8067-8075.

15 Yamamoto R, Nishikori M, Kitawaki T, et al. PD-1-PD-1 ligand interaction contributes to immunosuppressive microenvironment of Hodgkin lymphoma. Blood 2008;111:3220-3224.

16 Carreras J, Lopez-Guillermo A, Roncador G, et al. High numbers of tumor-infiltrating programmed cell death 1-positive regulatory lymphocytes are associated with 
improved overall survival in follicular lymphoma. J Clin Oncol 2009;27:1470-1476.

17 Muenst S, Hoeller S, Dirnhofer S, et al. Increased programmed death-1+ tumor-infiltrating lymphocytes in classical Hodgkin lymphoma substantiate reduced overall survival. Hum Pathol 2009;40:1715-1722.

18 Thompson RH, Dong H, Lohse CM, et al. PD-1 is expressed by tumor-infiltrating immune cells and is associated with poor outcome for patients with renal cell carcinoma. Clin Cancer Res 2007;13:1757-1761.

19 Loos M, Giese NA, Kleeff J, et al. Clinical significance and regulation of the costimulatory molecule B7-H1 in pancreatic cancer. Cancer Lett 2008;268:98-109.

20 Dong H, Strome SE, Salomao DR, et al. Tumorassociated B7-H1 promotes T-cell apoptosis: a potential mechanism of immune evasion. Nat Med 2002; 8:793-800.

21 Gao Q, Wang XY, Qiu SJ, et al. Overexpression of PDL1 significantly associates with tumor aggressiveness and postoperative recurrence in human hepatocellular carcinoma. Clin Cancer Res 2009;15:971-979.

22 Nomi T, Sho M, Akahori T, et al. Clinical significance and therapeutic potential of the programmed death-1 ligand/programmed death-1 pathway in human pancreatic cancer. Clin Cancer Res 2007;13:2151-2157.

23 Thompson RH, Kuntz SM, Leibovich BC, et al. Tumor B7-H1 is associated with poor prognosis in renal cell carcinoma patients with long-term follow-up. Cancer Res 2006;66:3381-3385.

24 Herait P, Ganem G, Lipinski M, et al. Lymphocyte subsets in tumour of patients with undifferentiated nasopharyngeal carcinoma: presence of lymphocytes with the phenotype of activated T cells. Br J Cancer 1987;55:135-139.

$25 \mathrm{Li}$ J, Zeng XH, Mo HY, et al. Functional inactivation of EBV-specific T-lymphocytes in nasopharyngeal carcinoma: implications for tumor immunotherapy. PLoS One 2007;2:e1122.

26 Yao M, Ohshima K, Suzumiya J, et al. Interleukin-10 expression and cytotoxic-T-cell response in EpsteinBarr-virus-associated nasopharyngeal carcinoma. Int J Cancer 1997;72:398-402.

$27 \mathrm{Xu}$ J, Menezes J, Prasad U, et al. Elevated serum levels of transforming growth factor beta1 in Epstein-Barr virus-associated nasopharyngeal carcinoma patients. Int J Cancer 1999;84:396-399.

28 Ho SY, Guo HR, Chen HH, et al. Prognostic implications of Fas-ligand expression in nasopharyngeal carcinoma. Head Neck 2004;26:977-983.

$29 \mathrm{Lau} \mathrm{KM}$, Cheng SH, Lo KW, et al. Increase in circulating Foxp3+CD4+CD25(high) regulatory $\mathrm{T}$ cells in nasopharyngeal carcinoma patients. Br J Cancer 2007;96:617-622.

30 Klibi J, Niki T, Riedel A, et al. Blood diffusion and Th1-suppressive effects of galectin-9-containing exosomes released by Epstein-Barr virus-infected nasopharyngeal carcinoma cells. Blood 2009;113: 1957-1966.

31 Blackburn SD, Shin H, Haining WN, et al. Coregulation of CD8+ $\mathrm{T}$ cell exhaustion by multiple inhibitory receptors during chronic viral infection. Nat Immunol 2009;10:29-37.

32 Nakamoto N, Kaplan DE, Coleclough J, et al. Functional restoration of HCV-specific CD8 T cells by PD-1 blockade is defined by PD-1 expression and compartmentalization. Gastroenterology 2008;134:1927-1937.

33 Karim R, Jordanova ES, Piersma SJ, et al. Tumorexpressed B7-H1 and B7-DC in relation to PD-1+ T-cell infiltration and survival of patients with cervical carcinoma. Clin Cancer Res 2009;15:6341-6347.

34 Oudejans JJ, Harijadi H, Kummer JA, et al. High numbers of granzyme B/CD8-positive tumour-infiltrating lymphocytes in nasopharyngeal carcinoma biopsies predict rapid fatal outcome in patients treated with curative intent. J Pathol 2002;198:468-475.

35 Heinecke L, Proud D, Sanders S, et al. Induction of B7-H1 and B7-DC expression on airway epithelial cells by the Toll-like receptor 3 agonist double-stranded RNA and human rhinovirus infection: In vivo and in vitro studies. J Allergy Clin Immunol 2008; 121:1155-1160.

36 Wang J, Cheng L, Wondimu Z, et al. Cutting edge: CD28 engagement releases antigen-activated invariant NKT cells from the inhibitory effects of PD-1. J Immunol 2009;182:6644-6647.

37 Wong RM, Smith KA, Tam VL, et al. TLR-9 signaling and TCR stimulation co-regulate CD8(+) T cell-associated PD-1 expression. Immunol Lett 2009;127:60-67. 